\title{
COMPARAÇÃO DO TEMPO DE LATÊNCIA ENTRE NOMEAÇÃO E LEITURA EM ESCOLARES ${ }^{1}$
}

Luciene Stivanin ${ }^{*}$

Cláudia Scheuer

\begin{abstract}
RESUMO. O objetivo desta pesquisa foi comparar o tempo de latência entre a leitura de palavras e a nomeação de figuras. Participaram 74 crianças, de $2^{\mathrm{a}}, 3^{\mathrm{a}}$ e $4^{\mathrm{a}}$ séries do Ensino Fundamental de escola pública, sem queixas de alterações no desenvolvimento e sem dificuldades escolares. Foram aplicadas provas de leitura e de nomeação, com estímulos equivalentes. O tempo de latência, referente ao tempo entre a apresentação do estímulo e o início da produção oral, foi registrado por meio de um programa computacional. Os resultados apontaram para um tempo de latência maior para a nomeação do que para a leitura, em todas as séries. A diferença entre as séries foi observada apenas na leitura de palavra. As características psicolingüísticas dos estímulos afetam o processamento de palavras e de figuras de forma diferente, porém estas tarefas apresentam um ponto em comum, mais evidente no que se refere à frequiência dos estímulos.
\end{abstract}

Palavras-chave: leitura, nomeação, tempo de latência.

\section{COMPARISON OF LATENCY TIME BETWEEN NAMING AND READING IN SCHOOL CHILDREN}

\begin{abstract}
Latency time between word reading and naming picture tasks is compared. Seventy-four children, grades 2,3 and 4 grades of a primary public school, without developmental complains and without schooling difficulties, participated. Word reading and naming picture with equivalent stimuli were applied. Latency time, registered in a computational program, was the period between stimuli presentation and the beginning of the child's oral response. Results show that children from all grades spend longer latency time for naming. Difference in grades was observed only for reading. Psycholinguistics traits of the stimuli affect in different manners the word and picture processing. Tasks have a common denominator which is more evident in stimuli frequency.
\end{abstract}

Key words: Word reading, picture naming, latency time.

\section{COMPARACIÓN DEL TIEMPO DE LATENCIA ENTRE NOMBRAMIENTO Y LECTURA EN ESCOLARES}

\begin{abstract}
RESUMEN. El objetivo de esta investigación fue el de comparar el tiempo de latencia entre la lectura de palabras y el nombramiento de figuras. Participaron 74 niños, de $2^{\underline{o}}, 3^{\text {er }}$ y $4^{\underline{0}}$ años del Enseñanza Primaria de escuela pública, sin quejas de alteraciones en el desarrollo y sin dificultades escolares. Fueron aplicadas pruebas de lectura y de nombramiento, con estímulos equivalentes. El tiempo de latencia, que se refiere al tiempo entre la presentación del estímulo y el inicio de la producción oral, fue registrado por medio de un programa computacional. Los resultados señalaron un tiempo de latencia mayor para el nombramiento, comparado al tiempo de latencia para la lectura, en todos los años. La diferencia entre los años fue observada sólo en la lectura de palabra. Las características psicolingüísticas de los estímulos afectan el procesamiento de palabras y de figuras en forma distinta, pero estas tareas presentan un punto en común, más evidente en lo que se refiere a la frecuencia de los estímulos.
\end{abstract}

Palabras-clave: Lectura, nombramiento, tiempo de latencia.

\footnotetext{
1 Apoio: Fapesp.

* Fonoaudióloga. Doutora em Ciências da Reabilitação.

\# Fonoaudióloga. Doutora em Lingüística. Docente do Curso de Fonoaudiologia, Departamento de Fisioterapia, Fonoaudiologia e Terapia Ocupacional da FMUSP.
} 
Esta pesquisa visa estudar a relação entre leitura de palavras isoladas e nomeação de figuras em escolares de $2^{\mathrm{a}}$ a $4^{\mathrm{a}}$ séries, em uma óptica cognitiva.

Vários estudos apontam para a relação entre a linguagem oral e a escrita. Valdois (2001) ressalta que um bom domínio da linguagem oral, tanto no âmbito da produção quanto no da compreensão, é importante para a criança em processo de aprendizagem da leitura.

Em fases precoces do desenvolvimento da linguagem, as crianças ouvem e percebem as palavras como única e inteira unidade e, ao falá-las, acessam sua representação fonológica como um todo. Sons similares parecem facilitar o crescimento do vocabulário. Crianças pequenas utilizam-se destas estratégias para estocar e memorizar palavras com sons similares mas com significados diferentes (faca/vaca; pente/dente). Posteriormente as estratégias de recuperação da palavra mudam: as crianças processam partes e não mais o todo das palavras, iniciando a construção de representações fonológicas e morfológicas, as quais, por sua vez, influenciam o desenvolvimento da leitura (Goswami, 2001; Hoskyn, 2004).

Para Goff, Pratt e Ong (2005), crianças com habilidades de linguagem mais desenvolvidas apresentam conhecimento mais extenso do significado das palavras e dos circuitos semânticos, utilizando-os para auxiliar na identificação na leitura da palavra escrita.

Desta forma, a presença de uma palavra no vocabulário oral da criança facilita-lhe a leitura da palavra correspondente, quando esta é vista pela primeira vez, levando à precisão e a um menor tempo de reconhecimento, por terem sido estabelecidas representações fonológicas e semânticas prévias (McKague, Pratt \& Johnston, 2001).

A relação entre a leitura de palavras e a nomeação de figuras tem sido investigada em crianças com dificuldades específicas no desenvolvimento da leitura, e as pesquisas apontam que o conhecimento semântico das palavras está intacto nestas crianças. Por outro lado, observa-se lentidão no acesso à informação fonológica, ocorrendo substituições de fonemas durante a nomeação (Faust \& SharfsteinFriedman, 2003; Fowler \& Swainson, 2004; Murphy, Pollatsek \& Well, 1988; Nation \& Snowling, 2001; Snowling, 1980; Swan \& Goswami, 1997; Troia, Roth $\&$ Yeni-Komshian, 1996).

A fim de se entender a relação entre nomeação e leitura, são descritos os modelos de processamento de figuras e de palavras escritas. Com relação à nomeação de figuras, inicialmente o objeto deve ser identificado como pertencente a uma classe particular de objetos, por sua aparência ou traçado, orientação e disposição. Então nomes apropriados devem ser ativados entre as palavras já conhecidas no léxico mental, e, finalmente, deve haver uma organização fonoarticulatória para que uma resposta específica possa ser executada (Johnson, Clark \& Paivio, 1996).

Um dos modelos utilizados para explicar como a informação escrita é processada é o de dupla rota, segundo o qual a leitura pode ser realizada por meio de um processo semântico-lexical ou por um processo fonológico (Coltheart, Curtis, Atkins \& Haller, 1993; Ellis, 1995; Lecours \& Parente, 1997). A rota semântico-lexical é usada para a leitura de uma palavra por meio da recuperação do seu significado e de sua pronúncia, já armazenados na memória; já a leitura por meio da rota fonológica envolve a conversão grafofonêmica diante de uma palavra não estocada no léxico (Coltheart et al., 1993; Ellis, 1995; Lecours \& Parente, 1997).

Quando se compara o processamento de figuras e o de palavras escritas, observa-se que as características ortográficas e fonológicas do estímulo são as que mais influenciam a leitura de palavras, enquanto as características semânticas (familiaridade, idade de aquisição) estão mais relacionadas à nomeação de figuras (Bates, Burani, D’Amico \& Barca, 2001).

No que se refere ao tempo envolvido na leitura e na nomeação, Theios e Amrhein (1989) explicam que, no caso de uma figura, a imagem é processada em um sistema gráfico e, logo após, ativa o sistema semântico. Após a figura ter sido identificada, um nome deve ser selecionado entre um número de nomes apropriados armazenados no léxico mental do sistema lingüístico, levando à produção da fala. Nessa óptica, a leitura de palavras isoladas pode ocorrer mais rapidamente do que a nomeação de figuras, diferença que se deve a dois processos extras necessários para a nomeação: o acesso ao significado da figura e a seleção do nome correto no léxico mental. Marmurek e Rinaldo (1992) afirmam, porém, que o tempo para o processamento dos estímulos envolvidos em cada tarefa (leitura e nomeação) muda com a idade e o desenvolvimento.

Devido às particularidades da ortografia de cada língua, as relações entre o desenvolvimento da leitura e outras habilidades de linguagem, como a nomeação, também diferem (Sprenger-Charolles, Cole, Béchennec \& Kipffer-Piquard, 2005; Vellutino, Fletcher, Snowling \& Scandlon, 2004; Zoccolotti et al., 2005).

Muitos estudos com crianças brasileiras envolvem a relação da leitura com o desenvolvimento da 
consciência fonológica (Cielo, 2002; Guimarães, 2003; Salles, Mota, Cechella \& Parente, 1999; Salles \& Parente, 2002) e com nomeação seriada rápida (Cardoso-Martins \& Pennington (2001). No que se refere à relação entre leitura e nomeação, Stivanin e Scheuer (2005) observaram, em um estudo de grupopiloto com crianças falantes do português do Brasil, que a nomeação de figuras e a leitura de palavras sofreram influência de variáveis psicolingüísticas no tempo de latência e na precisão. Nesta pesquisa, porém, não foi realizada a comparação entre tempo de latência para a nomeação de figuras e tempo de leitura de palavras isoladas.

O objetivo deste estudo foi comparar as provas de leitura de palavras isoladas e de nomeação de figuras com relação ao tempo de latência, levando em conta a escolaridade e as características psicolingüísticas dos estímulos.

As hipóteses deste estudo formulam um tempo de latência maior para a nomeação do que para a leitura em leitores de $4^{\text {a }}$ série; em crianças menos escolarizadas, o tempo para a nomeação é menor do que o tempo para a leitura; a nomeação de figuras e a leitura de palavras apresentam a influência das mesmas variáveis psicolingüísticas (freqüência, regularidade e extensão).

\section{MÉTODO}

Trata-se de um estudo observacional e transversal, aprovado pela Comissão de Ética para Análise de Projetos de Pesquisa do Hospital das Clínicas da Faculdade de Medicina da Universidade de São Paulo (protocolo de pesquisa $n^{0} 637 / 03$ ), para cuja realização foi assinado pelos responsáveis o competente termo de consentimento pós-informação do mesmo hospital.

\section{Casuística}

Os participantes desta pesquisa foram 74 crianças de $2^{\mathrm{a}}, 3^{\mathrm{a}}$ e $4^{\mathrm{a}}$ séries do ensino fundamental de uma escola regular e pública da cidade de São Paulo. Participaram crianças de ambos os sexos e com idades entre 7 e 12 anos.

Pais e professores forneceram informações sobre o desenvolvimento das crianças. Os sujeitos com queixas de alterações no desenvolvimento e com histórico de fracasso escolar, reprovação, evasão escolar e indicação de classe escolar foram excluídos do estudo antes da avaliação específica em leitura e nomeação. Durante a aplicação das provas específicas, as crianças que tiveram dificuldades na identificação da palavra escrita ou problemas de compreensão do teste e de trocas fonológicas também foram excluídas da pesquisa. As características dos sujeitos quanto à idade e gênero são apresentadas nas tabelas 1 e 2 .

Tabela 1. Distribuição dos Sujeitos por Idade.

\begin{tabular}{ccccc}
\hline & \multicolumn{4}{c}{ Séries } \\
\cline { 2 - 5 } $\mathbf{M}$ & $\mathbf{2}^{\mathbf{a}}$ & $\mathbf{3}^{\mathbf{a}}$ & $\mathbf{4}^{\mathbf{a}}$ & Total \\
\hline $\mathbf{D P}$ & $303,28^{*}$ & $114,58^{*}$ & $128,64^{*}$ & 115,51 \\
$\mathbf{n}$ & 25 & 5,78 & 7,89 & 12,11 \\
& 25 & 24 & 25 & 74 \\
\hline
\end{tabular}

Legenda: M-média, DP-desvio-padrão

* em meses

Tabela 2. Distribuição dos Sujeitos por Gênero.

\begin{tabular}{|c|c|c|c|c|c|c|c|}
\hline & & \multicolumn{6}{|c|}{ série } \\
\hline & & \multicolumn{2}{|r|}{$2^{a}$} & \multicolumn{2}{|c|}{$3 \mathbf{a}$} & \multicolumn{2}{|r|}{$4 a$} \\
\hline & & $\mathbf{n}$ & $\%$ & $\mathbf{n}$ & $\%$ & $\mathbf{n}$ & $\%$ \\
\hline \multirow{2}{*}{ gênero } & $\mathbf{F}$ & 13 & $52,0 \%$ & 12 & $50,0 \%$ & 14 & $56,0 \%$ \\
\hline & M & 12 & $48,0 \%$ & 12 & $50,0 \%$ & 11 & $44,0 \%$ \\
\hline \multicolumn{2}{|c|}{ Total } & 25 & $100,0 \%$ & 24 & $100,0 \%$ & 25 & $100,0 \%$ \\
\hline
\end{tabular}

Legenda: F- feminino; M- masculino

\section{Material e procedimentos}

Seleção da amostra: para a seleção utilizou-se uma anamnese, com o objetivo de obter dados sobre intercorrências pré-, peri e pós- natais, desenvolvimento neuropsicomotor, desenvolvimento auditivo e de linguagem, antecedentes familiares para alterações de linguagem e aprendizagem, comportamento e saúde em geral; e aplicou-se um questionário escolar com questões sobre o desempenho escolar, a fim de excluir as crianças que apresentassem fracasso escolar, repetência, evasão e necessidade de escola especial. A anamnese foi aplicada aos pais individualmente e o questionário escolar foi explicado aos professores, com a solicitação de que o preenchessem..

Utilizaram-se também, para exclusão/inclusão: o Questionário de Morbidades Psiquiátricas Infantis QMPI (Almeida Filho, 1985) para verificação de eventuais problemas comportamentais; o Questionário de Capacidades e Dificuldades- SDQ (Fleitlich, Cortazar \& Goodman, 2000) para verificação de problemas emocionais, comportamentais e sociais; e a Escala de Conners (Barbosa \& Gouveia,1993), para descartar problema atencionais e de hiperatividade. As informações foram dadas por pais e professores. As crianças que atingiram o ponto de corte para reprovação nas escalas foram excluídas.

Avaliação da leitura e da nomeação: as duas provas foram constituídas por estímulos equivalentes, isto é, na prova de leitura a palavra gaveta, e na prova 
de nomeação, a figura de uma gaveta. As provas de leitura e de nomeação foram aplicadas com intervalo de 15 dias entre uma e outra, para que não houvesse influência de uma prova em outra.

Prova de leitura: foram selecionadas 96 palavras escritas balanceadas por freqüência de ocorrência, extensão da palavra, complexidade silábica $e$ regularidade para o português do Brasil escrito.

Freqüiência: refere-se ao número de vezes em que as palavras escritas pertencentes ao vocabulário de uma língua ocorrem dentro de faixas etárias específicas. Foram selecionadas palavras de alta e baixa frequiências de ocorrência, obtidas do trabalho desenvolvido por Pinheiro (1996) com crianças na faixa pré-escolar e séries iniciais. Extensão da palavra: refere-se ao número de grafemas que compõem uma palavra. Foram considerados 4 e 6 grafemas para palavras de estrutura simples, e 5 ou mais grafemas para as palavras com estrutura complexa.

Complexidade silábica: refere-se à estrutura da palavra e à disposição dos grafemas nas sílabas. Os nomes simples são caracterizados por estrutura silábica CV (consoante-vogal) e os nomes complexos por $\mathrm{CV}$ e outras estruturas, como CCV (consoanteconsoante-vogal) e CVC (consoante-vogal-consoante). Não foram incluídos os dígrafos.

Regularidade para a leitura: diz respeito às regras de conversão grafema-fonema durante a leitura, caracterizando palavras regulares e irregulares. São palavras regulares aquelas que apresentam relação unívoca entre grafema e fonema, a qual pode ser independente ou dependente do contexto. Por outro lado, palavras irregulares são aquelas cujas relações grafofonêmicas são ambíguas. No português, apenas relações entre grafemas e fonemas são irregulares ( $<$ x $>$ : xadrez, fixo, máximo e exame; $<$ e $>$ e $<$ o $>$ em posição paroxítona tônica) (Lecours \& Parente, 1997).

As palavras escritas foram caracterizadas por letras impressas em negrito, fonte Microsoft Sans Serif, tamanho 40.

Prova de nomeação de figuras: as figuras equivalentes às palavras escritas foram selecionadas de um total de 400 figuras de Cycowicz, Friedman, Rothstein e Snodgrass (1997) e de sites de imagens na Internet (Google). As figuras foram constituídas por traçado preto e fundo branco, com tamanho de 5 x 5 $\mathrm{cm}$, aproximadamente.

\section{Programa computacional}

O programa para aplicação das provas foi criado especialmente para esta pesquisa por Pasquini (2004) no Departamento de Engenharia Eletrônica da
Universidade Estadual de Campinas (UNICAMP), por meio do sistema JAVA.

Os estímulos da prova de leitura e de nomeação foram apresentados isoladamente no centro da tela (15 polegadas) do computador. Foram inseridos 5 elementos (palavras escritas ou figuras) com o objetivo de oferecer explicações e treino para os sujeitos.

Para a aplicação de todas as provas, a criança foi posicionada de frente para a tela do computador e o microfone foi fixado à sua blusa. A criança foi instruída a dizer o nome da figura na prova de nomeação de figuras e a ler a palavra escrita na prova de leitura em voz alta de palavras isoladas. Após a resposta da criança, a pesquisadora apertava uma tecla para aparecer o estímulo seguinte.

Foram calculadas as médias do tempo de latência para cada palavra, grupo de estímulos e série e aplicados testes estatísticos paramétricos.

\section{RESULTADOS}

A análise estatística dos dados foi realizada por meio do teste ANOVA. No Gráfico 1 podem ser observadas as médias do tempo de latência para cada grupo de palavras em ambas as provas, nas três séries.

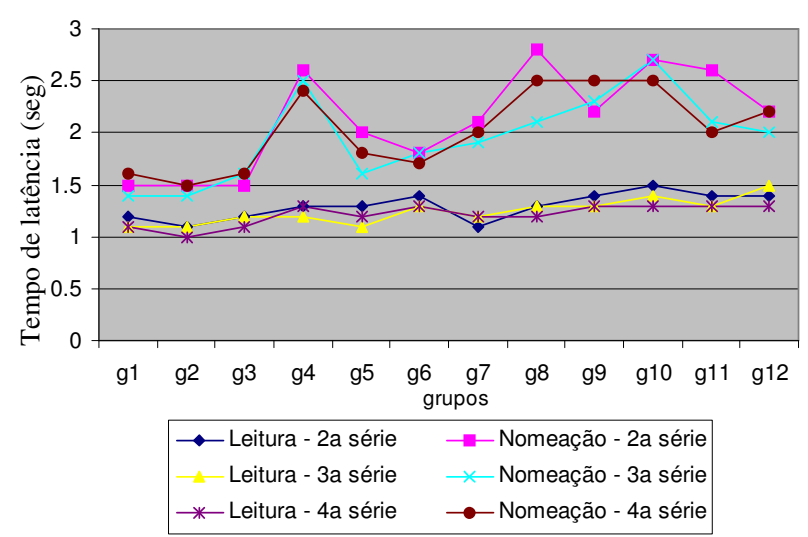

Legenda: g- grupos

g1- AF, 4G, CV, R; g2- AF, 4G, VC, I; g3- AF, 6G, CV R; g4- AF, 6G, CV I; g5- AF, 5 ou + G, OE, R; g6- AF, 5 ou +G, OE, I;

g7- $\mathrm{BF}, 4 \mathrm{G}, \mathrm{CV}, \mathrm{R}$; g8- BF, 4G, VC, I; g9- BF, 6G, CV R; g10- BF, 6G, CV I; g11- BF, 5 ou + G, OE, R; g12- BF, 5 ou +G, OE, I;

AF: alta freqüência; BF: baixa freqüência; G: grafemas; CV:consoante-vogal; OE: outras estruturas; $R$ : regular; I:irregular

Figura 1. Comparação do Tempo de Latência entre a Nomeação de Figuras e a Leitura de Palavras, nas Três Séries

O tempo de latência para a leitura de palavras foi menor para todos os tipos de palavra nas três séries. A diferença entre a leitura e a nomeação é maior, 
principalmente nos grupos de palavras de baixa freqüência.

$\mathrm{Na}$ Tabela 3, encontram-se os $p$-valores da comparação entre leitura e nomeação.

Tabela 3. Análise Estatística: Comparação do Tempo de Latência Entre as Provas de Nomeação e de Leitura.

\begin{tabular}{lccc}
\hline & \multicolumn{3}{c}{$\boldsymbol{p}$-valores } \\
\cline { 2 - 4 } $\begin{array}{l}\text { Características } \\
\text { psicolingüísticas }\end{array}$ & Série & Prova & Série x prova \\
\hline AF, 4G, CV, R & 0,4310 & $\mathbf{< 0 , 0 0 0 1} *$ & $<0,4168$ \\
AF, 4G, CV, I & 0,7814 & $<\mathbf{0 , 0 0 0 1} *$ & 0,3955 \\
AF, 6G, CV, R & 0,9908 & $<\mathbf{0 , 0 0 0 1} *$ & 0,6481 \\
AF, 6G, CV, I & 0,9217 & $<\mathbf{0 , 0 0 0 1} *$ & 0,8996 \\
AF, 5 e + G, OE, R & 0,0677 & $<\mathbf{0 , 0 0 0 1} *$ & 0,4984 \\
AF, 5 e +G, OE, I & 0,5487 & $<\mathbf{0 , 0 0 0 1} *$ & 0,7771 \\
BF, 4G, CV, R & 0,9873 & $<\mathbf{0 , 0 0 0 1} *$ & 0,5184 \\
BF, 4G, CV, I & 0,1782 & $<\mathbf{0 , 0 0 0 1} *$ & 0,1221 \\
BF, 6G, CV, R & 0,7823 & $<\mathbf{0 , 0 0 0 1} *$ & 0,3719 \\
BF, 6G, CV, I & 0,6132 & $<\mathbf{0 , 0 0 0 1} *$ & 0,9213 \\
BF, 5 e + G, OE, R & 0,2833 & $<\mathbf{0 , 0 0 0 1} *$ & 0,2718 \\
BF, 5 e +G, OE, I & 0,9183 & $<\mathbf{0 , 0 0 0 1} *$ & 0,4979 \\
\hline
\end{tabular}

$\mathrm{p}<0,05$

Legenda: AF: alta freqüência; BF: baixa freqüência; G: grafemas; CV:consoante-vogal; OE: outras estruturas; R: regular; I: irregular

Constata-se na tabela 3, na coluna prova, que os p-valores indicam diferença estatisticamente significante entre leitura e nomeação para todos os tipos de palavra, com o tempo de latência menor para a leitura do que para nomeação. Na coluna série, observa-se ausência de diferença significante entre as três séries estudadas, e na coluna série $\mathbf{x}$ prova os $\mathrm{p}$ valores indicam que a diferença entre ambas as provas ocorre da mesma forma em todas as séries, ou seja, tempo de leitura menor do que o de nomeação.

Para melhor entender as características da leitura e da nomeação, são descritos abaixo os dados referentes às características psicolingüísticas dos estímulos (freqüência, regularidade e extensão) e sua interação com a escolaridade, em cada prova.

Frequiência: na leitura de palavras, o tempo de latência foi menor para as palavras de alta freqüência do que para as de baixa $(p<0,05)$, em todos os tipos de palavra, com exceção das palavras mais simples (4G, $\mathrm{CV}$ e R) e mais complexas (5 e $+\mathrm{G}, \mathrm{OE}, \mathrm{I})$. Enquanto a $3^{\mathrm{a}}$ e a $4^{\mathrm{a}}$ séries apresentaram tempo de latência menor para palavras de alta frequiência e maior para as de baixa, as crianças de $2^{\mathrm{a}}$ série apresentaram o comportamento inverso. Na nomeação, observou-se que as figuras cujos nomes são mais freqüentes são nomeadas mais rapidamente do que aquelas com nomes pouco freqüentes $(p<0,001)$. Este dado oferece indícios da existência de relação entre a frequiência das palavras escritas e a das figuras.

Regularidade - na leitura, não houve efeito de regularidade no grupo de palavras de menor complexidade (AF, 4G, CV). Nos outros estímulos de alta freqüência, as palavras regulares foram lidas mais rapidamente do que as irregulares, por crianças de $2^{\mathrm{a}}$, $3^{\mathrm{a}}$ e $4^{\mathrm{a}}$ séries. Em todos os estímulos de baixa frequiência, o tempo de latência foi maior para a leitura das palavras irregulares do que das regulares apenas na $2^{\mathrm{a}}$ série, causando a interação série $\mathrm{x}$ palavra significante $(p<0,05)$. Na nomeação, o tempo de latência foi menor para nomes cuja escrita foi regular em apenas três dos grupos de estímulos (AF, 4G, CV; $\mathrm{BF}, 4 \mathrm{G}, \mathrm{CV}$; BF, 6G, CV).

Extensão: na leitura e na nomeação, o tempo de latência foi maior para as palavras mais extensas (6 grafemas) do que para as mais curtas (4 grafemas), em todas as séries $(p<0,05)$. As crianças das três séries apresentaram o mesmo comportamento $(p>0,05)$.

\section{DISCUSSÃO}

Os resultados obtidos apontaram diferenças entre o tempo de latência para a leitura e o tempo para a nomeação. A leitura apresentou menor tempo de latência do que a nomeação, independentemente das características psicolingüísticas dos estímulos. Este achado pode ser explicado pelo fato de a nomeação de figuras requerer sempre o acesso ao significado para a posterior produção do nome (Theios \& Amrhein, 1989; Johnson et al., 1996) e não depende de conversão grafofonêmica e da análise ortográfica (Trauzettel-Klosinski, Durrwachter, Klosinski \& Braun, 2006). Por outro lado, a leitura de palavras pode ser realizada sem passar por este processo, ou seja, a identificação de uma palavra escrita não armazenada no léxico ou pouco freqüente pode ser realizada por conversão grafema-fonema, sem a necessidade de acesso ao significado (Coltheart et al., 1993; Ellis, 1995; Lecours \& Parente, 1997). Neste processo, a informação semântico-lexical é ativada, mas não é um pré-requisito para iniciar a leitura (Roelofs, 2004).

Observou-se que, para todos os tipos de palavras de baixa frequiência, a diferença do tempo de latência entre leitura e nomeação foi maior, por ser maior o tempo para identificar as figuras. Isso pode ser justificado pelo fato que palavras menos freqüentes seriam preferencialmente lidas pelo processo fonológico, enquanto a nomeação das figuras equivalentes levaria mais tempo, por haver necessidade de acesso ao significado. 
$\mathrm{O}$ fato de os comportamentos das crianças de $2^{\mathrm{a}}$, $3^{\mathrm{a}}$ e $4^{\mathrm{a}}$ séries terem sido semelhantes quer dizer que todos os sujeitos do presente estudo apresentaram menor tempo de latência para a leitura. Estes achados não são os mesmos encontrados nas pesquisas de Marmurek e Rinaldo (1992). Estes autores demonstraram que universitários e alunos de $4^{\mathrm{a}}$ série lêem palavras mais rapidamente do que nomeiam os objetos correspondentes, enquanto o inverso ocorreu para os alunos de $2^{\mathrm{a}}$ série. Estes dados indicam que palavras e figuras não são necessariamente processadas pela mesma rota por todos os leitores, pois para leitores mais habilidosos, uma palavra pode ser lida antes de ocorrer o acesso ao seu significado.

Analisando cada prova quanto à influência das variáveis psicolingüísticas, observa-se o efeito de frequiência na leitura de palavras e na nomeação de figuras, em todas as séries. Tanto as palavras quanto as figuras mais freqüentes possuem representações consolidadas na memória e podem ser acessadas e recuperadas como um todo. Por outro lado, estímulos de baixa freqüência possuem um limiar de ativação maior e podem ser confundidos com outros visual ou fonologicamente semelhantes (Balota, Law \& Zevin, 2000; Coltheart, Rastle, Perry, Langdon \& Ziegler, 2001).

$\mathrm{O}$ efeito de freqüência, presente em ambas as provas, sugere uma relação entre as palavras e as figuras, já que o balanceamento realizado levou em conta apenas as características de palavras escritas. Como já relatado na introdução, o conhecimento de uma palavra influencia a facilidade com a qual ela é aprendida por uma criança em fase de desenvolvimento da leitura, pois suas representações de significado e de pronúncia estão consolidadas na memória e podem ser mais rapidamente recuperadas (McKague et al., 2001).

Quanto à regularidade, na prova de leitura as palavras regulares foram lidas mais rapidamente do que as irregulares. Este comportamento foi demonstrado pelas crianças de todas as séries, ao se considerarem os estímulos freqüentes. Nos estímulos de baixa freqüência, apenas a $2^{\mathrm{a}}$ série demonstrou diferença no tempo de latência quanto às palavras regulares e irregulares. Como em estágios iniciais de desenvolvimento da linguagem escrita a leitura é realizada mais frequientemente por conversão grafofonêmica (Salles \& Parente, 2002), a rota fonológica acarreta pronúncias conflitantes para as palavras irregulares; isso porque a leitura de palavras irregulares requer acesso ao léxico ortográfico e estas não podem ser lidas pela conversão grafofonêmica (Coltheart et al., 2001).
A leitura de palavras regulares e irregulares não foi semelhante à nomeação de figuras. Na nomeação de figuras, em apenas três grupos de estímulos houve diferença no tempo de latência. A regularidade se aplica somente a palavras escritas, e não a figuras, daí os resultados apontarem que, para este critério (regularidade), não há equivalência entre palavras e figuras. A nomeação é diretamente derivada do objeto representado, enquanto durante ou após a leitura é que o objeto se constitui mentalmente. Quando as palavras irregulares são lidas isoladamente, o tempo para reconhecê-las pode ser maior, pois a leitura deste tipo de palavra depende do contexto (frase ou sentença) para não gerar pronúncias conflitantes (/bolo/ e /bólo/).

No que diz respeito à extensão, as crianças de $2^{\mathrm{a}}$, $3^{\mathrm{a}}$ e $4^{\mathrm{a}}$ séries, apresentaram tempo de latência menor na leitura de palavras mais curtas $(\mathrm{CVCV})$ do que na das mais longas (CVCVCV). Este comportamento indica que quando palavras curtas são lidas mais rapidamente do que as longas a leitura está sendo realizada por meio de conversão grafofonêmica (Coltheart et al., 2001; Weekes, 1997). Enquanto na leitura de palavras o efeito de extensão foi observado em todos os grupos de estímulos, na nomeação de figuras este efeito ocorreu em dois dos quatro grupos de estímulos (associado a alta freqüência/irregular e baixa freqüência/regular). A explicação para a diferença entre os resultados da leitura e os da nomeação pode estar na utilização da rota de conversão grafofonêmica para a leitura e não para a nomeação.

Sintetizando, os efeitos de regularidade foram observados apenas na leitura de palavras, enquanto o de extensão e, principalmente, o de freqüência ocorreram para leitura e nomeação. Segundo Bates et al. (2001), a influência da ortografia e da fonologia é maior para a leitura de palavras, enquanto ambas as tarefas são afetadas pela frequiência, sugerindo que leitura e nomeação apresentam um componente lexical em comum.

Embora a nomeação de figuras tenha apresentado maior tempo de latência do que a leitura de palavras, para todos os grupos de estímulos, a influência das características psicolingüísticas foi diferente entre uma tarefa e a outra.

O objetivo deste estudo foi comparar o tempo de latência para a nomeação de figuras e a leitura de palavras. As hipóteses deste estudo foram confirmadas em parte. Retomando, uma das hipóteses se referia a um tempo de latência maior para a nomeação do que para a leitura em leitores de $4^{\mathrm{a}}$ série, enquanto em crianças menos escolarizadas, o tempo para a 
nomeação seria menor do que aquele para a leitura. Os resultados mostram que o tempo de latência para a nomeação foi maior do que para a leitura para todas as séries. Seria interessante comparar a leitura e a nomeação em crianças de $1^{\mathrm{a}}$ e a partir da $5^{\mathrm{a}}$ série para verificar se as diferenças no tempo de latência para a leitura e nomeação de figuras se modificam em função da escolaridade e de uma maior eficiência nestas habilidades ou se permanecem estáveis, por não interferirem mais no processo de aprendizagem de novas palavras.

Com relação à outra hipótese (a nomeação de figuras e a leitura de palavras apresentam a influência das mesmas variáveis psicolingüísticas), a leitura de palavras e a nomeação de figuras são afetadas diferentemente pelas variáveis psicolingüísticas, sendo o efeito de freqüência o que mais caracteriza a relação entre o processamento de palavras e o de figuras.

\section{CONCLUSÕES}

Nas crianças deste estudo, falantes do português do Brasil, o tempo de latência foi menor para a leitura do que para a nomeação, em todas as séries. Isto demonstra que a análise visual da figura e a necessidade de acesso ao significado para a adequada produção do nome são processos que levam mais tempo do que a análise ortográfica e/ou fonológica da palavra escrita, na faixa de escolaridade estudada.

A influência das características psicolingüísticas foi diferente para a leitura de palavras por efeitos de freqüência, regularidade e extensão, enquanto na nomeação, a freqüência foi a variável que mais exerceu influência. $\mathrm{O}$ fato de haver um componente lexical em comum para o processamento de palavras e de figuras sugere uma relação entre leitura e nomeação.

Este estudo sobre a relação entre linguagem oral (nomeação) e escrita (leitura) contribui para o desenvolvimento de estratégias para o processo de ensino-aprendizagem, na elaboração do material a ser utilizado e na escolha das atividades a serem empregadas.

\section{REFERÊNCIAS}

Almeida Filho, N. (1985). Epidemiologia das desordens mentais da infância no Brasil. Salvador: Centro Editorial e Didático da Universidade Federal da Bahia.

Balota, D. A., Law, M. B. \& Zevin, J. D. (2000). The attentional control of lexical processing pathways: Reversing the word frequency effect. Men Cognit, 28(7), 1081-1089.
Bates, E., Burani, C., D’Amico, S. \& Barca, L. (2001). Word reading and picture naming in Italian. Men Cognit, 29(7), 986999.

Bates, E., D’Amico, S., Jacobsen, T., Székely, A., Andonova, E. \& Devescovi, A. (2003). Timed picture naming in seven languages. Psychonomic Bulletin Review, 10(2), 344-80.

Barbosa, G. A. \& Gouveia, V. V. (1993). O fator hiperatividade do questionário de Conners: validade conceitual e normas diagnósticas. Temas, 38, 188-202.

Cielo, C. A. (2002). Habilidades em consciência fonológica em crianças de 4 a 8 anos de idade. Pró-fono Revista de Atualização Científica, 14(3), 301-312.

Cardoso-Martins, C. \& Pennington, B. F. (2001). Qual é a contribuição da Nomeação Seriada Rápida para Habilidades de Leitura e escrita? Evidência de Crianças e Adolescentes com e sem dificuldades de leitura. Psicologia: Reflexão e Crítica, 14(2), 387-397.

Coltheart, M., Curtis, B., Atkins, P. \& Haller, M. (1993). Models of reading aloud: Dual route and parallel - distributed processing approaches. Psychological Review, 100, 589-608.

Coltheart, M., Rastle, K., Perry, C., Langdon, R. \& Ziegler, J. C. (2001). DRC: A dual-route cascaded model of visual word recognition and reading aloud. Psychological Review, 108, 204-256.

Cycowicz, Y. M., Friedman, D., Rothstein, M. \& Snodgrass, J. G. (1997). Picture naming by young children: Norms for name, agreement, familiarity, and visual complexity. Journal of Experimental Child Psychology, 65,171-237.

Ellis, A.W. (1995, $2^{\mathrm{a}}$ ed.). Leitura e escrita e dislexia: uma análise cognitiva. Porto Alegre: Artes Médicas.

Faust, M. \& Sharfstein-Friedman, S. (2003). Naming difficulties in adolescents with dyslexia: Application of the tip-of-the-tongue paradigm. Brain and Cognition, 53(2), 211-217.

Fleitlich, B., Cortazar, P. G. \& Goodman, R. (2000). Questionáro de Capacidades e Dificuldades (SDQ). Infanto Revista de Neuropsiquiatria da Infância e Adolescência, 8, 44-50.

Frith, U., Wimmer, H. \& Lander, K. (1998). Differences in phonological recoding in German- and English- speaking children. Scientific Studies of Reading, 2, 31-54.

Fowler, A. E. \& Swainson, B. (2004). Relationships of naming skills to reading, memory, and receptive vocabulary: Evidence for imprecise phonological representations of words by poor readers. Ann Dyslexia, 54(2), 247-80.

Goswami, U. (2001). Early phonological development ant the acquisition of literacy. In S. B. Neuman \& D. K. Dickinson (Eds.), Handbook of Early Literacy Research (pp.11-125). New York: The Guilford.

Goff, D. A., Pratt, C. \& Ong, B. (2005). The relations between children's reading comprehension, working memory, language skills and components of reading decoding in a normal sample. Readind and Writing, 18, 583-616.

Goswami, U., Ziegler, J. C., Dalton, L. \& Schneider, W. (2003). Nonword reading across ortographics: How flexible is the choice of reading units? Applied Psycholinguistics, 24, 235247.

Guimarães, S. R. K. (2003). Dificuldades no desenvolvimento da lectoescrita: o papel das habilidades metalingüísticas. Psicologia: Teoria e Pesquisa, 19(1), 33-45. 
Hoskyn, M. (2004). Language Processes and Reading Disabilities. In B. Wong (Ed.), Learning about Learning Disabilities (pp. 93-131). USA: Elsevier Academic.

Johnson, C. J., Clark, J. M. \& Paivio, A. (1996). Cognitive Components of Picture Naming. Psychological Bulletin, 120(1), 113-39.

Lecours, A. R. \& Parente, M. A. M. P. (1997). Dislexia: implicações do Sistema de Escrita do português. São Paulo: Artes Médicas.

Marmurek, H. H. C. \& Rinaldo, R. (1992). The development of letters and syllable effects in categorization, reading aloud, and picture naming. Journal of Experimental Child of Psychology, 53, 277-299.

McKague, M., Pratt, C. \& Johnston, M. B. (2001). The effect of oral vocabulary on reading visually novel words: A comparison of the dual-route-cascated and triangle frameworks. Cognition, 80(3), 239-70.

Murphy, L. A., Pollatsek, A. \& Well, A. D. (1988). Developmental dyslexia and word retrieval deficits. Brain Lang, 35(1), 1-23.

Nation, K. \& Snowling, M. J. (2001). Phonological and semantic contributions to children's picture naming skill: Evidence from children with developmental reading disorders. Language and Cognitive Processes, 16, 241-259.

Pasquini, R. (2004) Elaboração do programa computacional para obtenção dos tempos de processamento nas tarefas de leitura de palavras de nomeação de figuras. Departamento de Engenharia Elétrica da Universidade Estadual de Campinas. (Texto não publicado)

Pinheiro, A. M. V. (1996). Contagem de freqüência de ocorrência de palavras expostas a crianças na faixa de pré-escola e séries iniciais. Software produzido pela Associação Brasileira de Dislexia- ABD.

Roelofs, A. (2004). Seriality of phonological encoding in naming objects and reading their names. Memory and Cognition, 32(2), 212-222.

Salles, J. F., Mota, H. B., Cechella, C. \& Parente, M. A. M. P. (1999). Desenvolvimento da Consciência Fonológica de crianças de primeira e segunda séries. Pró-Fono Revista de Atualização Científica, 11(2), 68-76.

Salles, J. F. \& Parente, M. A. M. P. (2002). Relação entre os processos cognitivos envolvidos na leitura de palavras e as habilidades de consciência fonológica em escolares. Pró-Fono Revista de Atualização Científica, 14(2), 175-86.
Snowling, M. J. (1980). Object Naming deficits in developmental dyslexia. Journal of Research in Reading. 11, 67-85.

Sprenger-Charolles, L., Cole, P., Béchennec, D. \& Kipffer-Piquard, A. (2005). French normative data on reading and related shills from EVALEC: A new computadorized battery of tests (end Grade1, Grade 2, Grade 3, and Grade 4). European Review Applied Psychology, 55(3), 157-186.

Stivanin, L. \& Scheuer, C. I. (2005). Tempo de latência e exatidão para a leitura e para a nomeação em crianças escolares: estudo piloto. Revista Educação e Pesquisa, 31(3), 425-433.

Swan, D. \& Goswami, U. (1997). Picture naming deficits in developmental dyslexia: The Phonological Representations Hypothesis. Brain Lang, 56, 334-53.

Theios, J. \& Amrhein, P. C. (1989). Theoretical analysis of the cognitive processing of lexical and pictorial stimuli: Reading, naming and visual and conceptual comparisons. Psychological Review, 96, 5-24.

Trauzettel-Klosinsk, S., Durrwachter, U., Klosinski, G. \& Braun, C. (2006). Cortical activation during word reading and picture naming in dyslexic and non-reading-impaired children. Clinical Neurophysiology (in press).

Troia, G. A., Roth, F. P. \& Yeni-Komshian, G. H. (1996). Word frequency and age effects in normally developing children's phonological processing. Journal of Speech, Language and Hearing Research, 39, 1099-108.

Valdois, S. (2001, $2^{\mathrm{a}}$ ed.). As grandes etapas da aprendizagem. In A. Van Hoult \& F. Estienne (Orgs.), Dislexias: descrição, avaliação, explicação e tratamento. Porto Alegre: Artmed.

Vellutino, F., Fletcher, J. M., Snowling, M. J. \& Scandlon, D. M. (2004). Specific reading disability (dyslexia): What have we learned in the past four decades? Journal of Psychology and Psychiatry, 45(1), 2-40.

Weekes, B. S. (1997). Differential effects of number of letters on words and nonword naming latency. Quarterly Journal of Experimental Psychology, 50(A), 439-56.

Zoccolotti, P., De Luca, M., Pace, E., Gasperini, F., Judica, A. \& Spinelli, D. (2005). Word length effect in early reading and in developmental dyslexia. Brain Lang, 93, 369-73.

Recebido em 04/04/06 Aceito em 05/07/07

Endereço para correspondência: Luciene Stivanin. Rua Teodoro Sampaio, 63 ap. 52, Pinheiros, CEP 05406-000, São Paulo-SP. E-mail: stivanin@usp.br 